\title{
Room-Temperature Electron Spin Amplifier Base on Ga(In)NAs Alloys
}

\author{
Yuttapoom Puttisong, Irina Buyanova, Aaron J. Ptak, C. W. Tu, Lutz Geelhaar, \\ Henning Riechert and Weimin Chen
}

\section{Linköping University Post Print}

N.B.: When citing this work, cite the original article.

This is the authors' version of the following published article:

Yuttapoom Puttisong, Irina Buyanova, Aaron J. Ptak, C. W. Tu, Lutz Geelhaar, Henning Riechert and Weimin Chen, Room-Temperature Electron Spin Amplifier Base on Ga(In)NAs Alloys, 2013, Advanced Materials, (25), 5, 738-742.

http://dx.doi.org/10.1002/adma.201202597

Copyright: Wiley-VCH Verlag Berlin http://www.wiley-vch.de/publish/en/

Postprint available at: Linköping University Electronic Press

http://urn.kb.se/resolve?urn=urn:nbn:se:liu:diva-85468 
DOI: 10.1002/adma.201202597

\section{Room-Temperature Electron Spin Amplifier Based on Ga(In)NAs Alloys}

By Y. Puttisong, I.A. Buyanova, A.J. Ptak, C.W. Tu, L. Geelhaar, H. Riechert, and W.M. Chen*

[*] Prof. W. M. Chen, Y. Puttisong, Prof. I.A. Buyanova, Department of Physics, Chemistry and Biology

Linköping University, S-581 83 Linköping (Sweden)

E-mail:wmc@ifm.liu.se

Dr. A.J. Ptak

National Renewable Energy Laboratory

Golden, Colorado 80401 (USA)

Prof. C.W. Tu

Department of Electrical and Computer Engineering

University of California, La Jolla, CA92093 (USA)

Dr. L. Geelhaar, Prof. H. Riechert

Paul-Drude-Institut für Festkörpelektronik

10117 Berlin (Germany)

Keywords: spin amplifier, spintronics, room temperature, defect, semiconductor

Efficient generation, maintaining, manipulation and detection of electron spin polarization and coherence at room-temperature (RT) in semiconductors is a prerequisite for the success of future semiconductor spintronics and quantum information technologies. ${ }^{[1-5]}$ Potential spintronic devices are expected to be based on fundamental building blocks such as spin filters (or spin injectors or spin aligners), spin amplifiers and spin detectors. During the past decade spin filters and spin detectors have been a main focal point of intense research efforts in the field of semiconductor spintronics that have led to many innovative approaches and encouraging developments. ${ }^{[1-5]}$ In sharp contrast, experimental developments in spin amplifiers have so far been extremely limited. This is despite of the facts that many theoretical proposals for a wide variety of model spin amplifiers have been suggested during the past decade, ranging from spin amplifiers using spin injection pillar ${ }^{[6,7]}$ or a dual-drain nonlocal lateral spin valve ${ }^{[8]}$ or non-magnetic semiconductors, ${ }^{[9]}$ unipolar spin transistors, ${ }^{[10]}$ 
magnetic dipolar transistors, ${ }^{[11,12]}$ spin gain transistors, ${ }^{[13]}$ and optically controlled spin transistors. ${ }^{[14]}$ As most of the proposed models require the involvement of a spin-functional diluted magnetic semiconductors (DMS), a lack of true DMS that can provide spin-polarized carriers at RT has so far prevented experimental realization of these proposed spin amplifiers. To our knowledge, the highest temperature at which a spin amplifier has so far been experimentally demonstrated to function is $150 \mathrm{~K}$ using a dual-drain nonlocal lateral spin valve based on an ferromagnetic metal-semiconductor hybrid structure. ${ }^{[8]}$

Here we propose and demonstrate a new concept of a defect-enabled spin amplifier based on a non-magnetic semiconductor (Figure 1a). We shall show that a large spin gain (up to $2700 \%$ ) can be obtained at RT by employing $\mathrm{Ga}(\mathrm{In}) \mathrm{NAs}$. This spin amplification functionality is facilitated by the spin-dependent recombination processes via defects, ${ }^{[15-17]}$ which was also recently explored for spin filtering. ${ }^{[15,18]}$ Spin amplification in the proposed spin amplifier is self-triggered by incoming conduction electrons with a finite spin polarization regardless of how weak and which direction the spin polarization is. Therefore, the sign of the spin polarization of the outgoing electrons from such a spin amplifier exactly follows that of the incoming electrons. Such spin amplification is generally efficient because even very weak spin polarization of incoming conduction electrons can dynamically polarize the spins of the defect electrons to a much greater degree, thanks to a much longer spin lifetime of the defect electrons commonly known in semiconductors. The spin-polarized defects can then in turn selectively capture and deplete conduction electrons with an opposite spin orientation (i.e. minority spin) governed by the Pauli exclusion principle, leading to greatly enhanced spin polarization of the conduction electrons. It should be pointed out that such spin amplification, or in other words amplification of magnetization, does not involve magnetism and no magnetic layers are required in this approach. Since there is no need to initialize and maintain the electron spin polarization of the defects by an external source other 


\section{ADNANCES
MAATRELAS}

than the input spin signal itself, the proposed spin amplifier is also expected to impose fewer technical restrictions in practical implementation in devices.

To demonstrate the effectiveness of the proposed spin amplification functionality, we employ an all-optical experimental method. Here, incoming conduction electrons of non-zero spin polarization are introduced by optical orientation ${ }^{[19]}$ under circularly polarized photoexcitation above the bandgap of $\mathrm{Ga}(\mathrm{In}) \mathrm{NAs}$. Spin polarization degree $\left(P_{e}\right)$ of the conduction electrons is measured by circular polarization degree $\left(P_{o}\right)$ of the band-to-band (BB) optical transitions, following their well-known correlation in semiconductors. The principle of the spin amplification should also apply in case of electrical spin injection and detection. Due to a general lack of reliable electrical spin detection at RT, however, we have chosen the optical method so that the effect of the spin amplification will not be obscured by complications arising from unknown and complex effects of poor electrical spin detection.

In Figure $1 \mathrm{~b}$ we display a typical photoluminescence (PL) spectrum obtained at RT from a GaInNAs layer. The PL band arises from the BB optical transitions from the conduction band (CB) to the valence band (VB) consisting of heavy-hole (hh) and light-hole (lh) state. The hh and lh states are degenerate in the studied GaInNAs as it is lattice matched to the GaAs substrate, whereas in the studied GaNAs epilayers and GaNAs/GaAs multiple quantum wells (MQW) the degeneracy is lifted due to an in-plane tensile strain field. The measured $P_{e}$ in the strain-free GaInNAs epilayer is also shown in Figure $1 \mathrm{~b}$, deduced from $P_{e}$ $=-2 P_{o}$ following the optical selection rules. Spin polarization of the CB electrons as high as $>40 \%$ can be obtained in this material thanks to the spin amplification, significantly higher than merely a few percents commonly observed in non-magnetic semiconductors limited by fast spin relaxation of $\mathrm{CB}$ electrons.

To confirm that the observed strong $P_{e}$ is indeed a result of the defect-enabled spin amplification, we have also studied $P_{e}$ in a reference sample of GaAs without the spinamplifying defects. Only $\sim 1 \% P_{e}$ was observed in such reference sample, see the black open 


\section{Submitted to

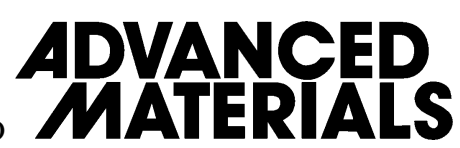

diamond symbols at zero field in Figure 1c, supporting the assignment. To further verify the defect-enabled spin amplification, we have closely examined depolarization effect of electron spins under a transverse magnetic field, known as the Hanle effect. This is motivated by the fact that the field strength required for complete spin depolarization due to Larmor precession of electron spins is determined by spin lifetime, which provides an effective means to separate contributions of $\mathrm{CB}$ and defect electrons due to their difference in spin lifetime. In a simplified picture commonly used in the literature, each individual depolarization process should give rise to a Hanle curve that can be described by a single Lorenzian line of which the half width is determined by the spin lifetime of the concerned electrons. Thus, contributions from two Lorenzian lines are expected in our case. This is confirmed by our experimental results, shown by the red open circles in Figure 1c from the GaInNAs epilayer. It clearly consists of two contributions, i.e. a sharp center line and a broad background. The sharp and dominant line, responsible for the quenching of the spin amplification effect and the resulting drastic drop of $\mathrm{CB}$ electron spin polarization (from $>40 \%$ to $\sim 2 \%$ ) within the field range of 0 $2000 \mathrm{G}$, corresponds to spin dephasing of the defect electrons with a longer spin lifetime. For CB electrons with a shorter spin lifetime, however, a larger field (thus a higher Larmor frequency) is required to cause spin dephasing within their spin lifetime (see the insert in Figure 1c). The background is thus contributed by the $\mathrm{CB}$ electron spin dephasing without the involvement of the spin amplification, indicated by the blue dashed line in Figure 1c. This assignment was further confirmed by the Hanle curve from the GaAs reference sample containing no spin-amplifying defects, which only exhibits the broad background as shown in Figure 1c by the black open diamond symbols. It is thus compelling from our experimental results that the defect-induced spin amplification is responsible for the observed strong $P_{e}$ at zero field in the GaInNAs sample.

Strictly speaking, a Hanle curve from a system with two coupled spin species like the $\mathrm{CB}$ and defect electron spins in the studied GaInNAs alloy cannot be simply deconvoluted 


\section{ADNANEDS
MATERRALIS}

into two Lorenzian components. A cascade spin depolarization process between the two spins must be taken into account. Therefore, to properly analyze the observed Hanle curves, we have employed coupled rate equations simultaneously describing the CB electron spin $(\vec{S})$ and the defect electron spin $\left(\vec{S}_{C}\right)$ as described in Supporting Information. A simulated Hanle curve from a best fit of the experimental data by the coupled rate equations is displayed by the green solid line in Figure 1c, showing an excellent agreement with the experimental data.

In order to unambiguously verify the spin amplification effect and to quantitatively determine the exact degree of the spin amplification, we have also simulated a Hanle curve in the absence of the spin amplification via the defects by setting $\vec{S}_{C}=0$ such that capture of $\mathrm{CB}$ electron by the defects is not spin selective. The result of the simulation is shown by the blue dash line in Figure 1c, which is strikingly different from the one involving the spin amplification (i.e. the solid green line) but is very similar to the Hanle curve measured from the GaAs reference sample without spin amplification (the black open diamond symbols). The disappearance of the sharp central component (highlighted by the grey shaded area in Figure 1c) confirms that the sharp component must arise from the defect-engineered spin amplification effect when $\vec{S}_{C} \neq 0$. It can also be clearly seen in Figure 1c that both green solid and blue dash line show nearly the same $P_{e}$ at high fields, where the defect-engineered spin amplification is completely quenched as a result of spin depolarization induced by the Larmor procession of the defect electrons in such high fields. Under such condition, the material returns to what is expected for an ordinary semiconductor such as the GaAs reference sample where $\mathrm{CB}$ electron spin polarization is mainly controlled by their fast spin relaxation. The spin amplification (SA) factor, i.e. spin gain, can therefore be obtained at zero field by $S A=P_{e}^{\exp } / P_{e}^{c a l}\left(\vec{S}_{C}=0\right) \quad, \quad$ or $\quad$ directly measured experimentally by $S A=P_{e}^{\exp }($ GaInNAs $) / P_{e}^{\exp }(G a A s)$. The obtained SA factor for the studied GaInNAs alloy is 


\section{ADNANCES
MATERRALI}

up to 27 (or 47) by using the first method (or the second method), which amplifies the CB electron spin polarization from merely $1.75 \%$ (or $1 \%$ ) to $>40 \%$ at RT !

It should be pointed out that the actual initial spin polarization degree of $\mathrm{CB}$ electrons generated by optical orientation is only $P_{e}^{i} \approx 1.75 \%$ in the GaInNAs sample and $P_{e}^{i} \approx 1 \%$ in the GaAs reference sample, which is remarkably lower than the theoretical value of $P_{e}^{i}=50 \%$ expected from the optical selection rule. This finding provides strong evidence for significant electron spin loss during optical excitation at high energy/momentum and subsequent energy/momentum relaxation from the excitation to emission photon energy, commonly seen in semiconductors. ${ }^{[20]}$ This finding further emphasizes the need for spin amplification, as spinpolarized carriers may often encounter energy relaxation during transport, e.g. in an applied electric field or across a hetero-interface.

We have performed similar experimental studies and detailed rate-equation analyses of the Hanle effect in all the samples studied in this work. The spin amplification effect is found to be general in all of the $\mathrm{Ga}(\mathrm{In}) \mathrm{NAs}$ alloys and their quantum structures, as long as the relevant defects have been introduced during the growth. The results from three representative samples are shown in Figure 2a-c, obtained by using $S A=P_{e}^{\exp } / P_{e}^{c a l}\left(\vec{S}_{C}=0\right)$. The SA values should be 1.75 times higher if they are estimated by $S A=P_{e}^{\exp }(G a I n N A s) / P_{e}^{\exp }(G a A s)$. Our results demonstrate that the spin amplification effect is universal in these dilute nitride alloys regardless of strain field (strained GaNAs vs strainfree GaInNAs lattice-matched to GaAs) or structures (epilayers vs MQWs). The observed strong dependence of the SA factor on excitation power reflects the corresponding dependence of the spin polarization degree of the defect electrons and thus the effectiveness of these defects in spin amplification. The observed difference in the SA factor among the studied samples is, on the other hand, mainly due to differences in the concentration of the spin-amplifying defects and also in the degree of initial $\mathrm{CB}$ electron spin polarization $P_{e}^{i}$ 


\section{Submitted to

generated by optical orientation, as a result of a different extent of spin loss during optical orientation and subsequent energy/momentum relaxation of photo-generated hot electrons. The latter is likely related to different concentrations of momentum and spin scattering centers as well as varying strengths of strain and built-in electric field, which critically depend on $\mathrm{N}$ compositions, structural design and growth conditions.

For an ideal spin amplifier, the amplified spin signal should truthfully follow the spinencoded information of the input spin signal that can be time-varying and can even switch the sign of spin polarization with time. To determine if our defect-enabled spin amplifier possesses such desired quality, we have studied in detail spin amplification of an electron spin signal that is modulated between positive and negative polarization by applying optical excitation of alternating circular polarization at $50 \mathrm{kHz}$. The results are shown in Figure 3a-b. The input spin signal without spin amplification is shown by the red dots in Figure $3 \mathrm{a}$ obtained at zero field from the GaAs reference sample, or by the black dots in Figure $3 b$ measured from $P_{e}$ at $2000 \mathrm{G}$ in GaInNAs that should be very close to the value of $P_{e}^{i}$ at zero field. The spin signal after the amplification was obtained by measuring $P_{e}$ at zero field in the GaInNAs sample, shown by the red open circles in Figure 3a-b. It can clearly be seen that the amplified spin signal has cloned the exactly same time-varying function of the input spin signal, or in other words it truthfully carries the same spin-encoded information, and at the same time it has substantially amplified the amplitude of the input signal. We have also performed simulations of the amplified spin signals based on the coupled rate equations (Equation 1 in Supporting Information) by using the same set of material parameters determined from the Hanle effect presented above. The simulated curves are displayed in Figure $3 a-b$ by the solid lines, in excellent agreement with the experimental data.

From Figure 1c, it is expected that the SA factor can be controlled between 27-1 by applying a weak field between 0-2000 G. This is also clearly demonstrated in the timevarying experiment by measuring $P_{e}$ in a field. The resulting experimental data at $200 \mathrm{G}$ are 
shown by the blue dots in Figure 3b, from which a reduction of the SA factor to about half of the maximum value can be estimated. Once again, the true form of the input spin signal is maintained after the spin amplification. Though an application of an external magnetic field is not a requirement for the spin amplification, it does offer an option for field control of the SA factor - another attractive feature of the proposed spin amplifier.

The response time, or the highest operation frequency, is an important quality factor of a spin amplifier, just as that for a current amplifier in conventional charge-based electronics. Unfortunately we were not able to experimentally determine the cut-off frequency of our spin amplifier as it is beyond the instrumental limitation of the fastest optical-polarization modulators and detectors commercially available in the concerned near infrared spectral range. Therefore, we have carried out simulations with the aid of the coupled differential equations (Equation 1 in Supporting Information). By using the same set of the parameters that have provided excellent fit to the experimental data from the Hanle effect (Figure 1c) and excitation power dependence (Figure 2a), we have systematically simulated the spin amplified signal under a modulation of the input spin signal at various frequencies. The simulated curves at $0.1 \mathrm{GHz}$ are shown in Figure 3c, as an example. They clearly confirm that the proposed defect-engineered spin amplifier remains fully functional at such a high frequency. A slight phase shift between the input and the amplified signal indicates that the selected modulation time starts to approach the response time of the spin amplifier. To estimate the cut-off frequency of the spin amplifier, we have calculated the peak spin polarization value as a function of modulation frequency of the input spin signal. The results are displayed in Figure 3d, which shows that the cut-off frequency of our spin amplifier is about $1 \mathrm{GHz}$ defined when the SA factor reduces to one half of its maximum value at the lower frequencies.

In summary, we have proposed and demonstrated a new type of spin amplifiers, namely a defect-engineered spin amplifier, based on a non-magnetic semiconductor. It represents the first experimental demonstration of a spin amplifier successfully operating at RT - a long- 


\section{Submitted to

waited missing fundamental building block in semiconductor spintronics. A large spin amplification factor (up to $\geq 27$ ) can be achieved at RT for $\mathrm{CB}$ electrons in $\mathrm{Ga}(\mathrm{In}) \mathrm{NAs}$ epilayers and GaNAs/GaAs MQWs, without requiring a magnetic layer. Such spin amplifier is shown to maintain the exactly same time-varying function and thus the spin-encoded information of an input spin signal, while substantially amplifying the amplitude of the input spin signal. It is demonstrated to be capable of amplifying fast-varying spin signals without distorting the wave form, with a predicted cut-off frequency of about $1 \mathrm{GHz}$. In addition, the spin gain can be easily tuned by controlling a weak magnetic field between 0 and $2000 \mathrm{G}$ leading to a controlled SA factor between the maximum value to 1 . In principle, such a spin amplifier can be incorporated in any place along a path of spin transport to enhance spin signals that have suffered spin loss during the transport. Its applications are also expected to reach beyond the straight-forward amplification when it is combined with other spintronic components. By amplifying spin polarization with such a spin amplifier, even a poor spin injector (or aligner) with a low efficiency could be sufficient to provide complete electron spin polarization and could potentially become a perfect spin injector. Likewise, by combining such a spin amplifier with a spin detector, a significant enhancement in spin detection efficiency could be achieved making possible to detect very weak spin signals. Applications of such spin amplifiers could thus potentially allow more tolerance for imperfect spin injector/detectors and also provide an alternative and viable solution to the current and important issue on RT spin injection and detection in semiconductors.

\section{Supporting Information}

Supporting Information is available from the Wiley Online Library or from the author.

\section{Acknowledgements}

This work was supported by Linköping University through the Professor Contracts, Swedish Research council (Grant No. 621-2011-4254), Swedish Energy Agency, and Knut and Alice Wallenberg Foundation. 
Submitted to

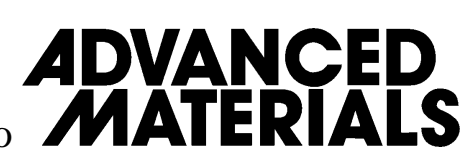

Received: ((will be filled in by the editorial staff))

Revised: ((will be filled in by the editorial staff))

Published online: ((will be filled in by the editorial staff))

[1] S. A. Wolf, D. D. Awschalom, R. A. Buhrman, J. M. Daughton, S. von Molnár, M. L. Roukes, A. Y. Chtchelkanova, and D. M. Treger, Science 2001, 294, 1488

[2] I. Žutić, J. Fabian, and S. Das Sarma, Rev. Mod. Phys. 2004, 76, 323.

[3] D. D. Awaschalom and M. E. Flatté, Nat. Phys. 2007, 3, 153.

[4] Spintronics, Semiconductors and Semimetals Vol.82, edited by T. Dietl, D.D. Awschalom, M. Kaminska and H. Ohno (Academic Press, New York, 2008).

[5] Handbook of Spintronic Semiconductors, edited by W. M. Chen and I. A. Buyanova (Pan Stanford, Singapore, 2010).

[6] Y. Acremann, X. W. Yu, A. A. Tulapurkar, A. Scherz, V. Chembrolu, J. A. Katine, M. J.Carey, H. C. Siegmann, and J. Stöhr, Appl. Phys. Lett. 2008, 93, 102513.

[7] G. Chin and J. Yeston Editor's Choice. A model spin amplifier, Science, 2008, 322, 17.

[8] D. Saha, M. Holub and P. Bhattacharya , Appl. Phys. Lett. 2007, 91, 072513.

[9] S-W. Jung and H-W. Lee, Phys. Rev. B 2006, 73, 165302.

[10] M. E. Flatté and G. Vignale. Appl. Phys. Lett. 2001, 78, 1273-1275.

[11] J. Fabian, I. Zutic' and S. Das Sarma, Phys. Rev. B 2002, 66, 165301.

[12] M. E. Flatté, Z. G. Yu, E. Johnston-Halperin, D. D. Awschalom, Appl. Phys. Lett. 2003, 82, 4740 .

[13] D. E. Nikonov and G. I. Bourianoff, IEEE Trans. Nanotechnology 2005, 4, 206-214.

[14] H-F. Lu, G. Yong, X-T. Zu and H-W. Zhang, Appl. Phys. Lett. 2009, 94, 162109.

[15] X. J. Wang, I. A. Buyanova, F. Zhao, D. Lagarde, A. Balocchi, X. Marie, C. W. Tu, J. C. Harmand and W. M. Chen, Nat. Mater. 2009, 8, 198.

[16] V. K. Kalevich, E. L. Ivchenko, M. M. Afanasiev, A. Yu. Shiryaev, A. Yu. Egorov, V. M. Ustinov, and Y. Masumoto, JETP Lett. 2005, 82, 455. 
[17] D. Lagarde, L. Lombez, X. Marie, A. Balocchi, T. Amand, V. K. Kalevich, A. Shiryaev, E. Ivechenko and A. Egorov, Phys. Stat. Sol. a 2007, 204, 208.

[18] Y. Puttisong, X. J. Wang, I. A. Buyanova, H. Carrére, F. Zhao, A. Balocchi, X. Marie, C. W. Tu, and W. M. Chen, Appl. Phys. Lett. 2010, 96, 052104.

[19] Optical Orientation, edited by F. Meier and B. P. Zakharchenya, (North-Holland, Amsterdam, 1984).

[20] W. M. Chen, I. A. Buyanova, K. Kayanuma, Z. H. Chen, A. Murayama, Y. Oka, A. A. Toropov, S. V. Sorokin, S. V. Ivanov and P. S. Kop'ev, Appl. Phys. Lett., 2004, 85, 5260.

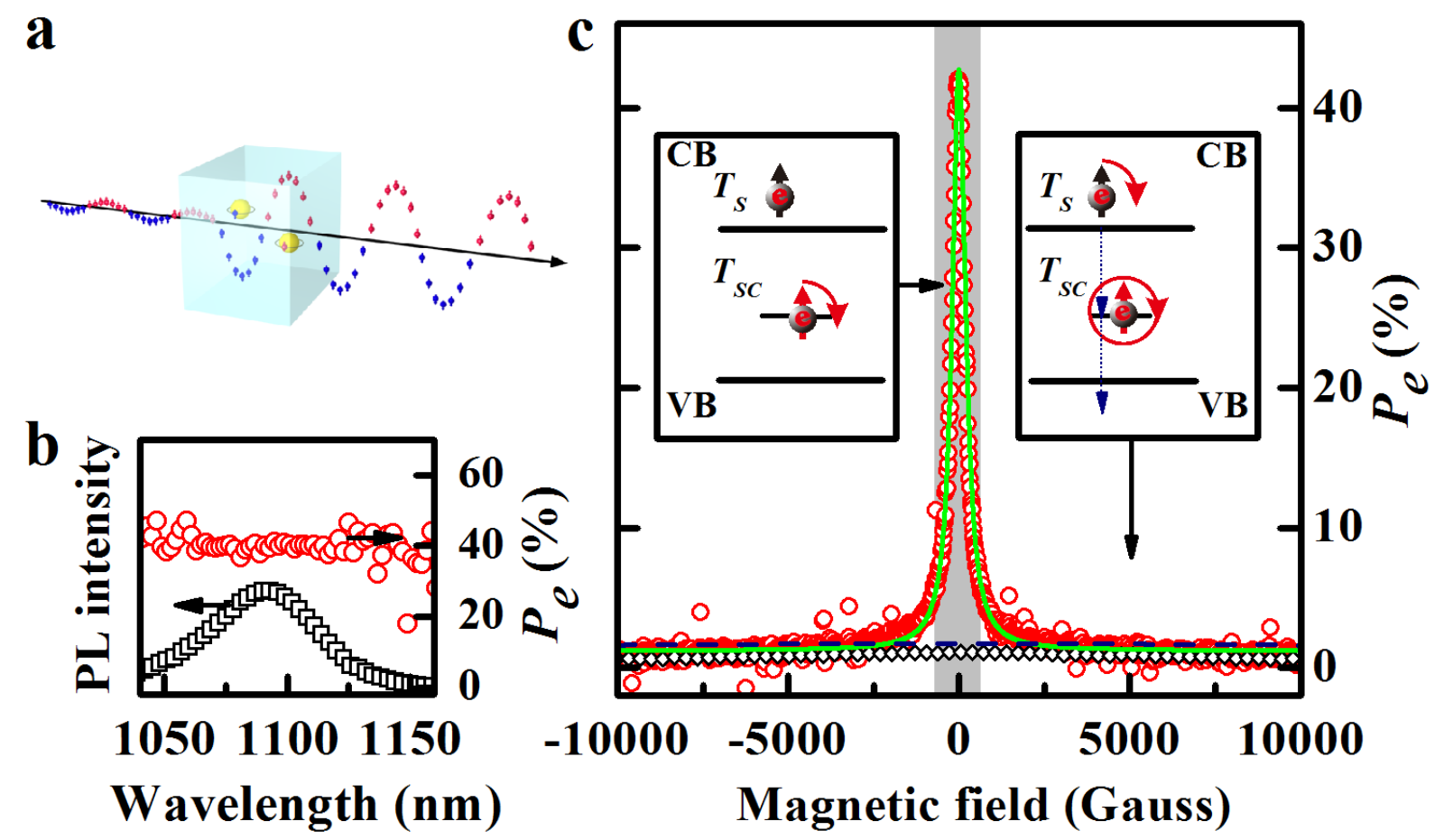

Figure 1. RT defect-enabled spin amplification as demonstrated by optical orientation and Hanle experiments. (a) A schematic picture of the defect-engineered spin amplifier demonstrated in this work. Suitable defects leading to the spin amplification in Ga(In)NAs are Ga interstitials. ${ }^{[15]}$ (b) Typical spectra of the BB PL and CB electron spin polarization at RT from the strain-free $\mathrm{Ga}_{0.96} \mathrm{In}_{0.04} \mathrm{~N}_{0.014} \mathrm{As}_{0.986}$ epilayer, obtained under $\sigma^{-}$circularly polarized excitation at the wavelength of $960 \mathrm{~nm}$ and with excitation power of $300 \mathrm{~mW}$. (c) A typical Hanle curve (the red open circles) obtained at RT by monitoring at the peak position of the PL band from the same sample shown in (b), under $\sigma^{-}$excitation at $925 \mathrm{~nm}$ and $440 \mathrm{~mW}$. The green solid and blue dashed lines are simulated Hanle curves with and without the spin 
Submitted to

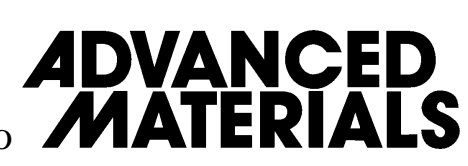

amplification effect, respectively, obtained from the coupling rate equations (Equation 1 in Supporting Information). The grey shaded area emphasizes the field range where the spin amplifier is functional, whereas beyond that the spin amplification is strongly suppressed by spin depolarization of the defect electrons due to Larmor precession (schematically shown by the inserts). The Hanle curve obtained at RT from the GaAs reference sample is shown by the black open diamond symbols, under $\sigma^{-}$excitation at $760 \mathrm{~nm}$ and $400 \mathrm{~mW}$.
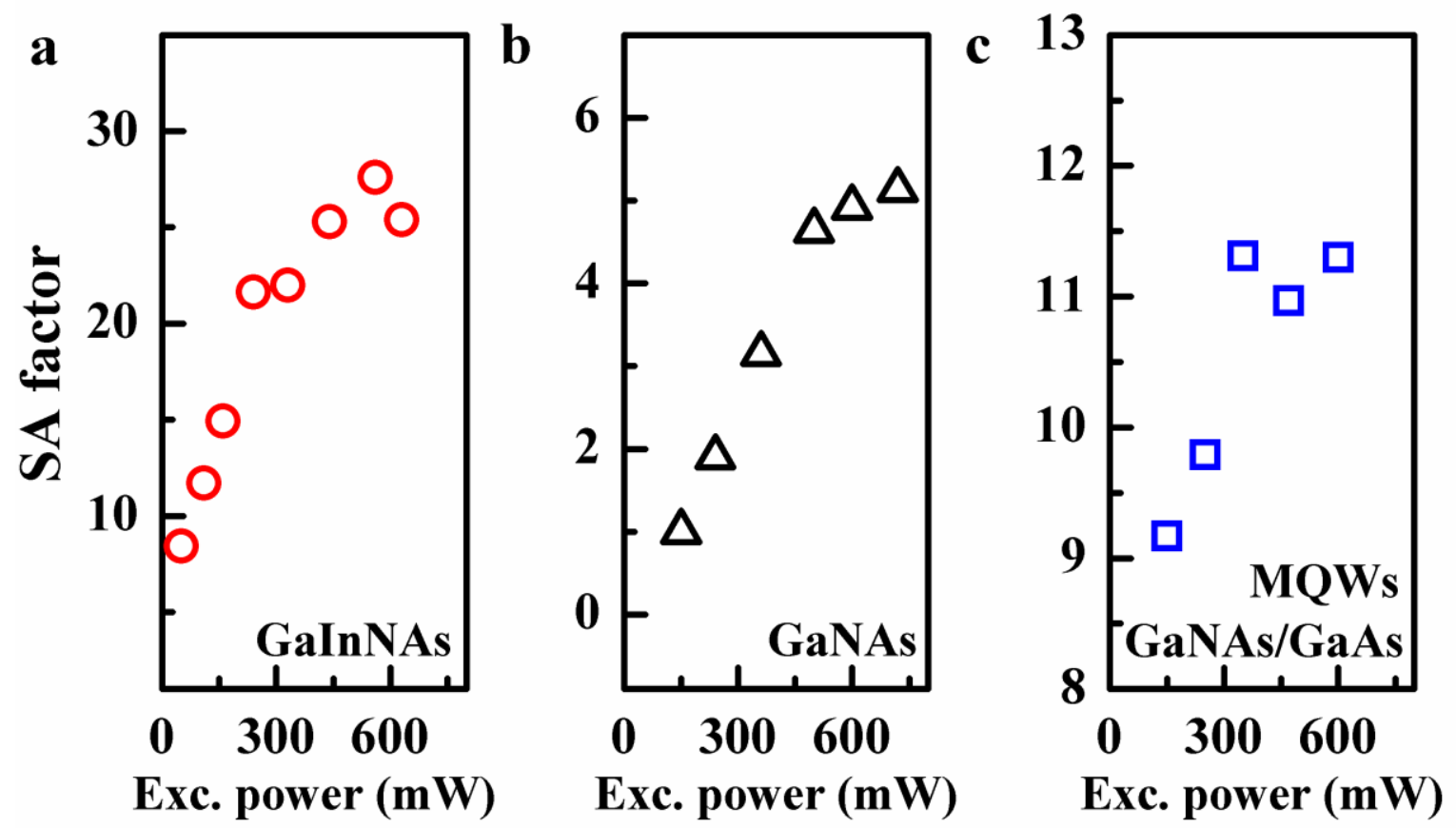

Figure 2. Measured spin amplification factors as a function of excitation power. SA factors as a function of excitation power obtained at RT from (a) the strain-free $\mathrm{Ga}_{0.96} \mathrm{In}_{0.04} \mathrm{~N}_{0.014} \mathrm{As}_{0.986}$ epilayer, (b) the strained $\mathrm{GaN}_{0.026} \mathrm{As}_{0.974}$ epilayer, and (c) the strained $\mathrm{GaN}_{0.016} \mathrm{As}_{0.984} / \mathrm{GaAs} \mathrm{MQW}$. The SA factors were obtained by detecting the PL maximum for (a) and the e-hh transition for (b-c). All data were collected with circularly polarized excitation at $925 \mathrm{~nm}$. 

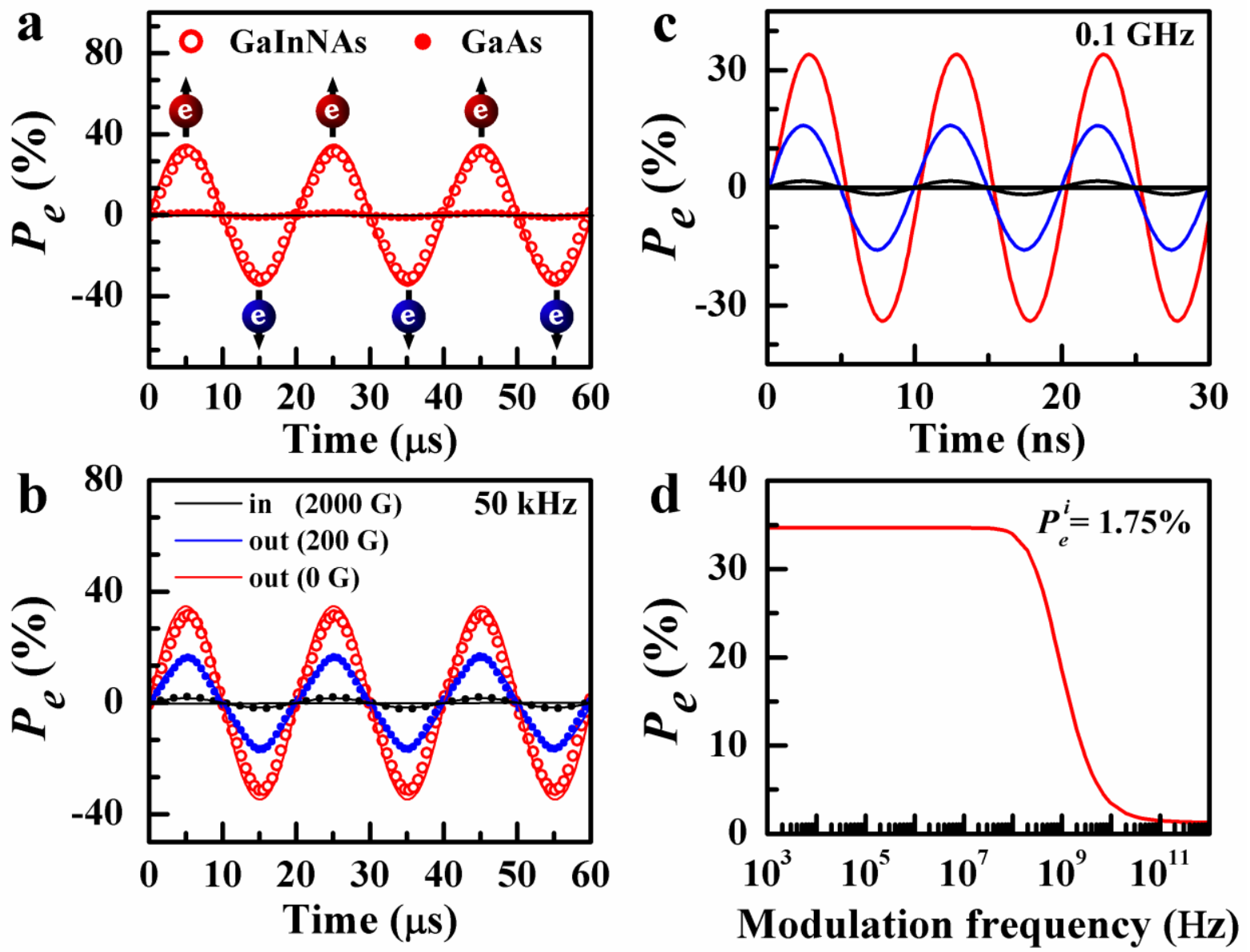

Figure 3. Frequency response of the spin amplifier. (a) Experimentally determined time variations of $P_{e}$ obtained at RT and zero field from the GaInNAs sample (the red open circles) and the GaAs reference sample (the red dots), under the alternating circularly polarized excitation at $50 \mathrm{kHz}$. The red solid line is a simulated spin-amplified signal. (b) Control of spin amplification by tuning external magnetic field. The input spin signal (the black dots) were measured at $2000 \mathrm{G}$ when the spin amplification is inactive. The spin amplified output signals obtained at 0 and $200 \mathrm{G}$ are denoted by the red open circles and the blue dots, respectively. The simulated input (the black line) and output spin signals (the red line at zero field and blue line at $200 \mathrm{G}$ ) are also shown. (c) Simulated time variations under the alternating circularly polarized excitation at a modulation frequency of $0.1 \mathrm{GHz}$. The black curve is the input spin signal, whereas the red and blue curves correspond to the amplified spin signal at 0 and $200 \mathrm{G}$, respectively. (d) Simulated peak value of $P_{e}$ as a function of the modulation frequency of the input spin signal, revealing the frequency responses of the spin amplifier. The simulations in (a)-(d) are obtained from Equation 1 in Supporting Information, by using the same parameters as deduced from the Hanle curves. 


\section{The table of contents entry:}

Keyword: spin amplifier, spintronics, room temperature, defect, semiconductor

By Y. Puttisong, I.A. Buyanova, A.J. Ptak, C.W. Tu, L. Geelhaar, H. Riechert, and W.M. Chen*

\section{Room-Temperature Electron Spin Amplifier Based on Ga(In)NAs Alloys}

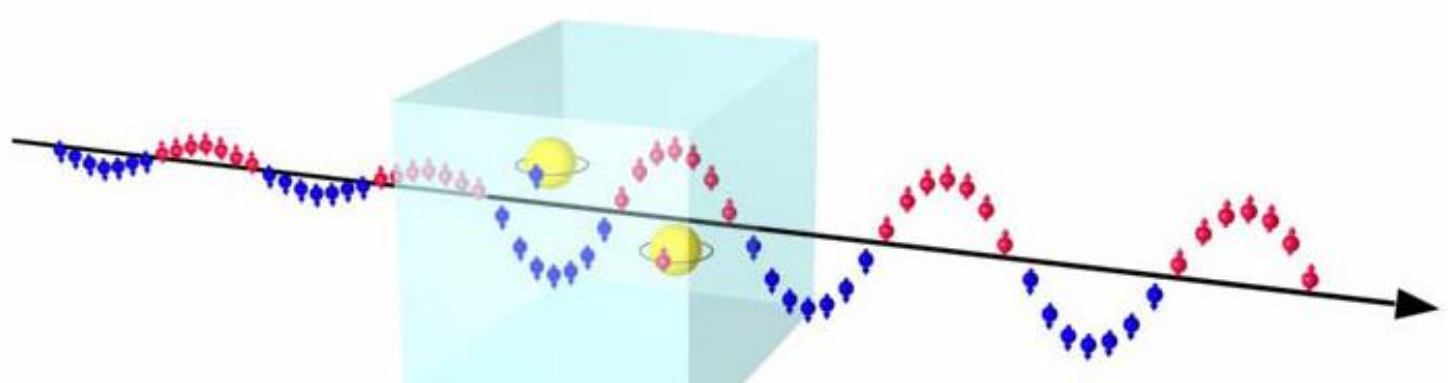

The first experimental demonstration of a spin amplifier at room temperature is presented. We propose and demonstrate an efficient, defect-enabled spin amplifier based on a non-magnetic semiconductor $\mathrm{Ga}(\mathrm{In}) \mathrm{NAs}$, with a large spin gain (up to $2700 \%$ at zero field) for conduction electrons and a high cut-off frequency up to $1 \mathrm{GHz}$. 


\section{Supporting Information:}

\section{Room-Temperature Electron Spin Amplifier Based on Ga(In)NAs Alloys}

By Y. Puttisong, I.A. Buyanova, A.J. Ptak, C.W. Tu, L. Geelhaar, H. Riechert, and W.M. Chen*

\section{Coupled rate equation analysis}

To properly analyze the observed Hanle curves from the studied $\mathrm{Ga}$ (In)NAs samples, we have employed coupled rate equations simultaneously describing the CB electron spin $(\vec{S})$ and the defect electron $\operatorname{spin}\left(\vec{S}_{C}\right)$ as follows: ${ }^{[1,2]}$

$$
\begin{aligned}
& \frac{d n}{d t}=G-\frac{\gamma_{e}}{2}\left(n N_{1}-4 \vec{S} \cdot \vec{S}_{C}\right), \\
& \frac{d \vec{S}}{d t}=\frac{G}{2} \vec{P}_{e}^{i}-\frac{\gamma_{e}}{2}\left(\vec{S} N_{1}-n \vec{S}_{C}\right)-\frac{\vec{S}}{\tau_{S}}-\vec{S} \times \vec{\Omega}, \\
& \frac{d \vec{S}_{C}}{d t}=-\frac{\gamma_{e}}{2}\left(n \vec{S}_{C}-\vec{S} N_{1}\right)-\frac{\vec{S}_{C}}{\tau_{S C}}-\vec{S}_{C} \times \vec{\Omega}_{C}, \\
& \frac{d p}{d t}=G-\gamma_{h} p N_{\uparrow \downarrow}, \quad N_{C}=N_{1}+N_{\uparrow \downarrow} .
\end{aligned}
$$

Here $G$ is the generation rate of electron $(n)-$ hole $(p)$ pair. $\vec{P}_{e}^{i}$ denotes initial spin polarization of CB electrons generated by optical orientation. $N_{1}$ and $N_{\uparrow \downarrow}$ are concentrations of the defects in the charge states with one and two bound electrons, respectively, whereas $N_{C}$ is the total concentration. $\vec{\Omega}=g \mu_{B} \vec{B} / \hbar$ and $\vec{\Omega}_{C}=g_{C} \mu_{B} \vec{B} / \hbar$ are Larmor frequencies of the $\mathrm{CB}$ electrons and the defect electrons, respectively, where $g$ and $g_{C}$ are their g-factors. $\gamma_{e}$ and $\gamma_{h}$ are the electron and hole capturing coefficients to the defects. $\tau_{S}$ and $\tau_{S C}$ are the spin relaxation times of the $\mathrm{CB}$ and defect electrons, respectively. The $\mathrm{BB}$ radiative carrier recombination is neglected in Equation 1 as it is more than two orders of magnitude slower than the capture and recombination via the defects. The analysis of the results shown in Figure 1c yields $\tau_{s}=140 \mathrm{ps}$ and $\tau_{s c}=520 \mathrm{ps}$. The parameters of $\gamma_{e} / \gamma_{h}=4$ 


\section{ADNANEDS
MATERRALIS}

and $\gamma_{e} N_{C}=1.14 \mathrm{ps}^{-1}$ were determined from excitation power dependence of $P_{e}$ and the spin amplification (Figure 2a) and $g_{C}=2$ and $g=1$ from earlier studies. ${ }^{[3,4]}$ The same parameters were used in the simulations shown in Figure 3.

\section{Experimental}

Samples: The Ga(In)NAs alloys investigated in this work were grown by molecular beam epitaxy (MBE) techniques at $390-540{ }^{0} \mathrm{C}$ on (001) GaAs substrates. The following three representative samples were studied, i.e. thin films of strained $\mathrm{GaN}_{0.026} \mathrm{As}_{0.974}(0.1 \mu \mathrm{m})$, strainfree $\mathrm{Ga}_{0.96} \mathrm{In}_{0.04} \mathrm{~N}_{0.014} \mathrm{As}_{0.986}(3 \mu \mathrm{m})$ alloys, and 7 periods of MQWs of $\mathrm{GaN}_{0.016} \mathrm{As}_{0.984} / \mathrm{GaAs}$ (90/200 ̊). As a reference sample without the defect-induced spin amplification, a $1 \mu \mathrm{m}$ thick, strain-free GaAs epilayer grown by MBE on a GaAs substrate was also studied.

Optical orientation and Hanle experiments: In optical orientation and Hanle measurements, a tunable Ti:Sapphire laser was employed as an excitation source with the laser beam propagating along the growth direction of the alloys. The excitation wavelength was 925-960 nm, i.e. with excitation energies above the $\mathrm{Ga}(\mathrm{In}) \mathrm{NAs}$ band gap. For the GaAs reference sample, the excitation wavelength was $760 \mathrm{~nm}$. Continuous-wave and alternating circular polarization of the excitation beam was created by a $\lambda / 4$ waveplate and a photoelastic modulator (PEM) operating at $50 \mathrm{kHz}$, respectively. The resulting PL signal was detected in a back-scattering geometry by a cooled Ge-detector integrated with a monochromator. PL polarization $P_{o}=\left(I^{+}-I^{-}\right) /\left(I^{+}+I^{-}\right)$was measured by using a PEM operating at $50 \mathrm{kHz}$ together with a linear polarizer. An external magnetic field in the range of -10000-10000 Gauss was applied in the Hanle experiments, with the field axis perpendicular to the growth direction of the alloys. All measurements were carried out at RT.

Supporting References: 


\section{Submitted to

[1] Optical Orientation, edited by F. Meier and B. P. Zakharchenya, (North-Holland, Amsterdam, 1984).

[2] E.L. Ivchenko, V. K. Kalevich, A. Yu. Shiryaev, M. M. Afanasiev and Y. Masumoto, J. Phys: Condens. Matter 2010, 22, 465804.

[3] X. J. Wang, I. A. Buyanova, F. Zhao, D. Lagarde, A. Balocchi, X. Marie, C. W. Tu, J. C. Harmand and W. M. Chen, Nat. Mater. 2009, 8, 198.

[4] V. K. Kalevich, E. L. Ivchenko, M. M. Afanasiev, A. Yu. Shiryaev, A. Yu. Egorov, V. M. Ustinov, and Y. Masumoto, JETP Lett. 2005, 82, 455. 